\title{
Design And Realization Of A Glass-Curtain Wall-Cleaning Robot
}

\author{
Qichao Zhao ${ }^{1}$, Xin Wang*, Jun Wang ${ }^{2}$, Huafeng Wen ${ }^{2}$,Jingwei Jiang ${ }^{1}$, Qingpei Zhang ${ }^{1}$ \\ 1.Harbin Institute of Technology Shenzhen Graduate School, Shenzhen, \\ 518055, China \\ 2.Shenzhen Fine Automatic Machine Co.,Ltd, Shenzhen \\ 518055 ,China
}

Key words : Glass-curtain wall-cleaning; Obstacle-crossing strategy; Dynamics; Adams simulation.

\begin{abstract}
With the extensive application of glass curtain wall in the city's high-rise buildings, the cleaning task becomes more and more heavy. This paper designed a glass curtain wall cleaning robot. The robot itself does not have walking mechanism, but it can move on smooth glass surfaces depending on the lifting fore of the trolley crane on the building roof while adhering to the glass curtain using the thrust of the propellers, calculate the wall inclination range that the robot can adapt according to the size of the propeller thrust. In cleaning process, it can take different actions to cross the obstacles, such as horizontal window frames, according to different height of them. And Adams simulation analysis is carried out for the process of crossing the higher obstacle. Wireless remote control is used in this robot control system.
\end{abstract}

\section{Introduction}

With the development of urban modernization and the increasing population, there are more and more high-rise building, and most outer surfaces of high-rise buildings are constructed with glass curtain walls. So the cleaning task becomes more and more heavy. At present, the high-rise glass-curtain wall is still cleaned by traditional manual cleaning methods ${ }^{[1]}$. This method, on the one hand, is low efficiency, on the other hand, workers' safety is difficult to be protected. Up to now, automated methods for cleaning high-rise glass-curtain walls have not been satisfactorily solved. In addition, the long-term exposure to the natural environment, glass-curtain wall will be burst. And if burst glass can't be found in time, some dangerous situations, such as falling glass will be occur. Most of the glass-curtain wall surfaces are flat, only some formed obstacles, such as glass window frame, exist, so the cleaning robot is the ideal choice for cleaning work. The use of cleaning robot will greatly improve the working environment of workers, and labor productivity, and has great social and economic significance and broad application prospects.

Since 1966, the universities and institutions at home and abroad started their research on the wall climbing robot. The earliest wall climbing robot was designed and realized ${ }^{[1]}$ by Xiliang of Osaka Prefecture University based on the principle that the pressure on the air inlet side of the fan is smaller than that on the air outlet side and the negative pressure can provide the adsorption capacity to the robot.

In 1978, A Japanese Chemical and Mechanical Technology Joint-stock Company developed a wall climbing robot, named as "Walker" [2]. The single sucking disc is applied in the robot and adsorption capacity comes from the negative pressure generated by a vacuum pump. It walks with the help of running rollers and two running belt and makes a turn through the speed difference between the rollers on right and left sides and the belt. The adsorption capacity of the wall climbing robot developed by the University of Miyazaki Japan comes from the thrust ${ }^{[3]}$. This robot doesn't need the sucking disc to generate adsorption capacity, but with the help of aerotechnics, the robot can firmly adsorb the wall surface through the thrust generated by propeller. The angle change of propeller's supporting arm enable it to move. And it has a comparatively strong ability in obstacle crossing. The robot "Robug" ${ }^{[4]}$ designed by University of Portsmouth England has spider-like legs with sucking disc to move on the wall surface. In 1986, the American International Robot Company developed a wall climbing robot "Skywasher" [5] for the cleaning of the skyscraper surface. Its movement is realized by the alternative adsorption of two groups of L-type frame sliding relatively. There are three soles in each frame group and two vacuum sucking disc on each sole. The sucking disc can help to make a rectilinear motion surface and also transverse motion relative to the wall to step over an obstacle with certain height. Our first independently developed wall-climbing robot is finished in research laboratory of Harbin Institute of Technology and it is developed for nuclear power application ${ }^{[6]}$. Since 1996, Beijing University of Aeronautics and Astronautics has successively developed a series of prototypes ${ }^{[7]}$ of glass curtain wall cleaning robot, like WASHMAN, CLEANBOT and SKYCLEAN. Shanghai University has successively developed a series of wall climbing robots with multi-frame and multiple vacuum sucking discs. The internal and external frame can make rectilinear motion relatively to the middle frame, and the robot can make right and left motion through the alternative usage of internal and external sucking discs ${ }^{[8]}$.

Shanghai Jiaotong University developed a robot with dual sucking disc. The cleaning robot carries a water tank and its adsorption capacity comes from the negative pressure of the sucking disc. There is no mechanism for movement in the robot, and with the help of the auxiliary mechanism on the building roof and its gravity it can realize the movements ${ }^{[9]}$. 
The City University of Hong Kong developed an air-driven wall climbing robot with cross form frame structure. This robot system is composed of movable climbing robot, auxiliary trolley, air compressor and controlling computer. The main body of the robot is composed of two perpendicular cylinders, whose stretching out and drawing back help the robot to move on all sides ${ }^{[10]}$.

Now, the glass-curtain wall-cleaning robot is still on the test and research stage at home and abroad and there is still a distance of road ahead until the real application. This paper will introduce a glass-curtain wall-cleaning robot which can be applied to wall surface with multiple inclination and window frame of different height. This paper will introduce the overall solution, the mechanical structure of the glass-curtain wall-cleaning robot and how to step over the obstacles during its working in the first part. And in the second part, the paper will present the analysis about force conditions and its kinetic model when the robot moves across surface with different angels. Motion control design and Adams simulation are described in the third section. Finally, conclusions and discussion of the results are drawn in the last section of the paper.

\section{Mechanical Structure of Cleaning Robot}

\subsection{The structure of robot system}

To achieve the glass curtain wall cleaning, the following components are necessary:

(1)The stable adsorption capacity on glass surfaces;

(2)The ability of moving on the glass surface;

(3)Having cleaning actuator.

The adhesion methods adopted by wall-climbing described in the previous section can be divided into three categories:

(1)Magnetic adsorption(including permanent magnets and electromagnet);

(2)Vacuum negative pressure adsorption;

(3)Thrust sorption simulating the propulsion principle of aircraft propulsion ${ }^{[10]}$.

The cleaning robot designed by this paper uses the thrust generated by the rotation of four propellers to make the robot adsorb to the wall for cleaning. In terms of movement, the robot itself does not have walking mechanism, and the movement is finished by an auxiliary movement mechanism installed at the top of the building, the body of the robot is linked to it with a steel cable, and the auxiliary movement mechanism itself can move horizontally. The up-and-down movement of the cleaning robot is realized by the retracting of the wire rope, and the left-right movement is realized by the lateral movement of the auxiliary movement mechanism. The overall structure of the system is shown in Figure1. The cleaning executive structure of the robot is composed of a roller brush and two flexible scrapers. The robot relies on the rolling friction between the roller brush driven by brushless DC motor and the glass wall to clean the stain. The mechanical structure of the glass-curtain wall-cleaning robot is shown as Figure 2. And the three - dimensional model of the cleaning robot is shown as figure 3 .

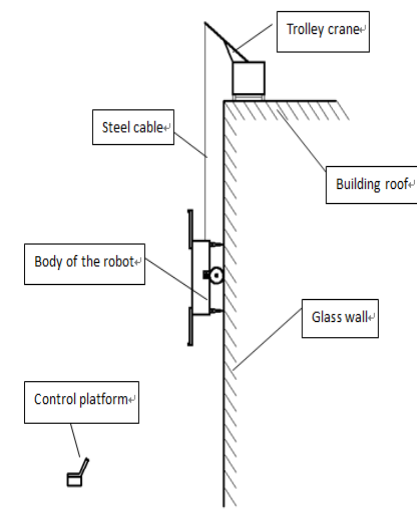

Figure 1: The overall structure of the system

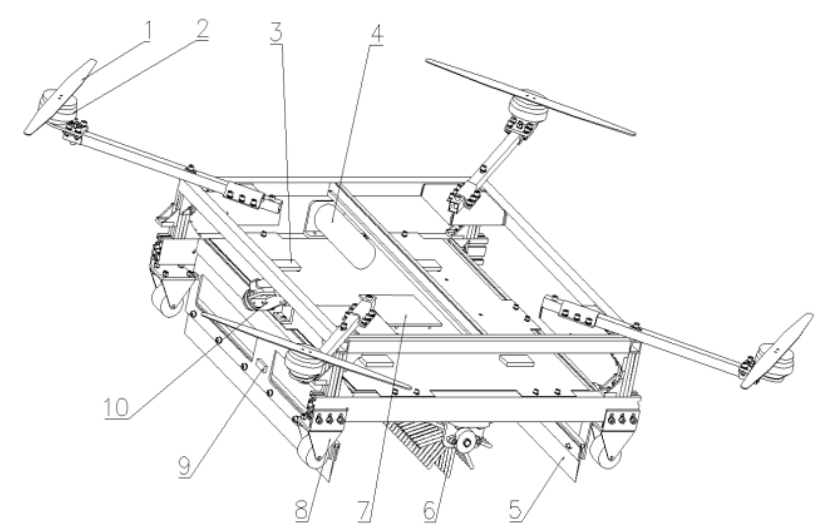

1.blade of propeller 2.propeller drive motor 3.electronic governor 4.brushless DC motor 5.flexible scraper 6.roller brush 7.STM32 control board 8. collision avoidance wheel 9. ultrasonic sensor 10.camera sensor

Figure 2: Mechanical structure of the glass-curtain wall-cleaning robot

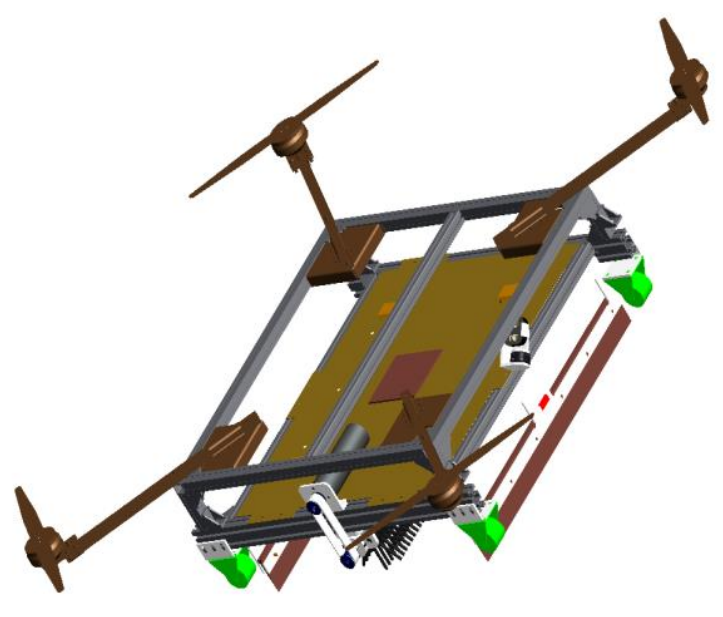

Figure 3:The three - dimensional model of the robot

\subsection{The strategy of crossing the obstacle}

Cleaning robot will encounter obstacles such as glass window frames while cleaning glass, the height of the protruding 
window frame is different as the glass wall surface structure is different. So the robot will take different actions to cross the obstacles according to different height of the window frame. The roller brush and two scrapers are all flexible, and the maximum deformation is $3-4 \mathrm{~cm}$. They will be deformed under the thrust of propellers during the cleaning work, when the height of window frame is lower than $4 \mathrm{~cm}$, the cleaning robot can move across the window frame by the deformation of roller brush and two scrapers.

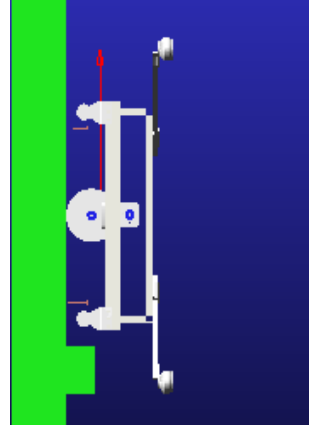

a: stop moving when approaching the fram

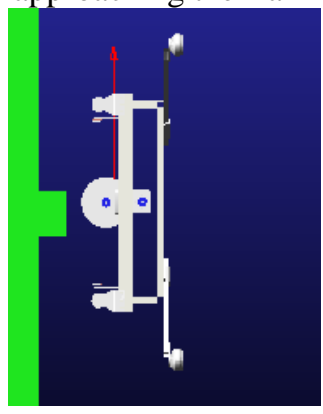

c: stepping over the obstacle

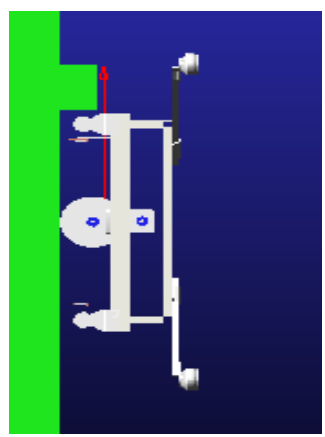

e: moving back to the surface

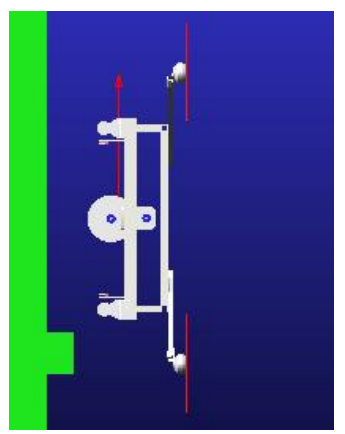

$\mathrm{b}:$ Departure from the wall surface

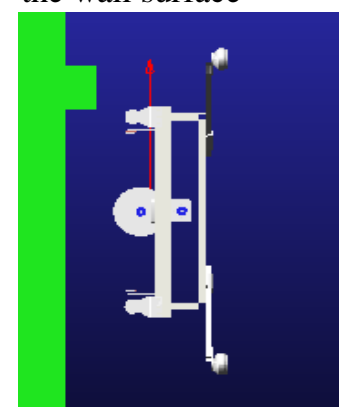

d: stepping over the obstacle

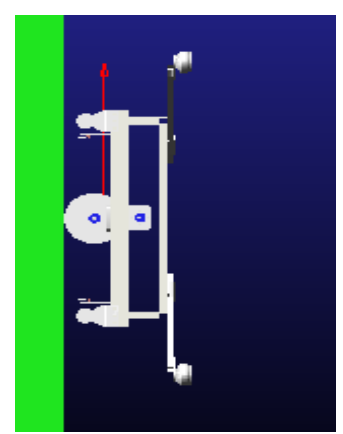

f: continue to clean
Figure 4: Obstacle crossing process of the glass-curtain wall-cleaning robot

But when the height of window frame is higher than $4 \mathrm{~cm}$, cleaning robot can't cross the frame in this way, in this case the steps of crossing obstacles are as follows:

(1)When the cleaning robot approaches the window frame, the auxiliary movement mechanism stops the cleaning robot from moving up and down.

(2)The propellers first stop and then reverse so that the propellers produce a thrust perpendicular to the wall outward.
(3)Adjust the propellers' speed to change the size of the thrust to make the robot away from the wall a certain distance.

(4)The robot moves continuously and crossed the obstacles

(5)Reduce the propellers' speed to make the cleaning robot reattached back to the wall.

(6)The robot continues cleaning work.

In this case, the crossing steps are shown in Figure 4.

\section{Dynamic Analysis of cleaning robot}

\subsection{Analysis of Forces under different inclination of glass wall}

The thrust generated by the propellers are all used to provide the suction force when the cleaning robot works on a vertical wall, however, when the wall has a certain inclination, part thrust generated by the propellers is used to overcome the component of robot's gravity while cleaning robot working or crossing obstacles. If the inclination is too large, the propellers may not meet the thrust required by the robot for normal operation. In the following, the range of the wall's inclination to be adapted to the cleaning robot is analyzed. The wall's inclination can be divided into two cases, one is inclined along the vertical wall inward, and the other is outward. Suppose that the thrust generated by four propellers is $\mathrm{T}_{1}, \mathrm{~T}_{2}, \mathrm{~T}_{3}, \mathrm{~T}_{4}$, total thrust $\mathrm{T}=\mathrm{T}_{1}+\mathrm{T}_{2}+\mathrm{T}_{3}+\mathrm{T}_{4}$. The weight of the cleaning robot body is $\mathrm{G}$, in the case where the wall is inclined along the vertical wall inward is shown as Figure 5 a ,

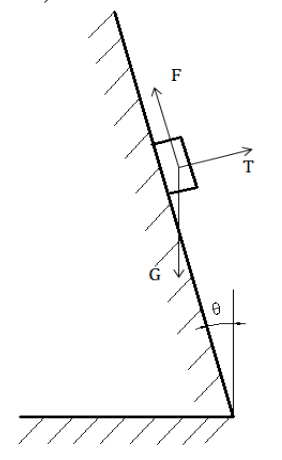

a: inward tilt

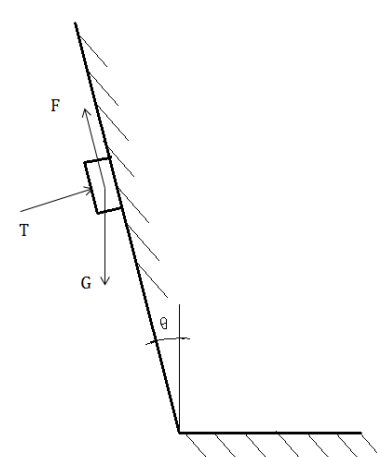

b: outward tilt
Figure 5: Two cases of the wall

When the cleaning robot crosses the higher window frame obstacle, the propeller thrust should satisfy the following formula:

$$
\mathrm{T}>\mathrm{G} \sin \theta
$$

$\theta$ is the angle between the glass wall and the vertical surface, thus, the maximum inclination can be deduced:

$$
\theta_{\text {max }}=\sin ^{-1} \frac{\mathrm{T}_{\max }}{\mathrm{G}}
$$

And in the case where the wall is inclined along the vertical wall outward is shown as Figure $5 \mathrm{~b}$, in addition to overcoming the part force of cleaning robot's gravity, the propellers also provide s certain thrust to ensure a certain positive pressure between the wall and cleaning robot. Suppose that the positive pressure cleaning robot needed for 
normal working is $\mathrm{N}$, the propeller thrust should satisfy the following formula:

$$
\mathrm{T}>\mathrm{N}+\mathrm{G} \sin \theta
$$

Thus, the maximum inclination can be deduced:

$$
\theta_{\max }=\sin ^{-1} \frac{\mathrm{T}_{\max -\mathrm{N}}}{\mathrm{G}}
$$

\subsection{The dynamical model analysis on the cleaning robot}

To ensure the robot can step over the higher window frames, we need to analyze the posture of the robot when it is stepping over the obstacle. First we need to set up the coordinate system for the robot, and define the ground coordinates $\mathrm{E}$ and carrier coordinates $\mathrm{B}$, both right-handed coordinates, shown as Figure 6.

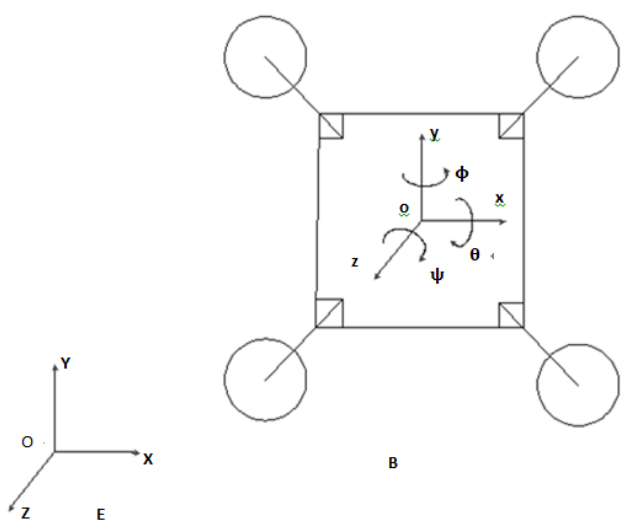

Figure 6: Coordinate system of the robot

And then we need to define the position and posture of the robot in the ground coordinates, while the conversion of the vectorial coordinate got from the robot sensors between these two coordinates need to be realized by the rotation matrix ${ }^{[12]}$. We assume in the ground coordinates, the origin coordinate of the robot is $\mathrm{T}^{\mathrm{E}}=\left[\begin{array}{lll}\mathrm{x} & \mathrm{y} & \mathrm{z}\end{array}\right]$, its dip angle is $\theta^{\mathrm{E}}=\left[\begin{array}{lll}\theta & \varnothing & \varphi\end{array}\right], \varnothing$ is the angle of pitch; $\theta$ is the roll angle and $\varphi$ is the yaw angle additional, the vector quality in the robot coordinates is

$$
a_{B}=\left[\begin{array}{lll}
x_{B} & y_{B} & z_{B}
\end{array}\right]
$$

and it is converted in the ground coordinate system to be :

$\mathrm{R}$ is the rotation matrix,

$$
a_{E}^{\prime}=\mathrm{R} * a_{B}=\left[\begin{array}{lll}
x_{E} & y_{E} & z_{E}
\end{array}\right]
$$

$$
\mathrm{R}=
$$

$\left[\begin{array}{ccc}\cos \psi \cos \theta & \cos \psi \sin \phi-\sin \psi \cos \theta \quad \cos \psi \sin \theta \cos \phi+\sin \psi \sin \phi\end{array}\right.$ $\left[\begin{array}{ccc}\sin \psi \cos \theta & \sin \psi \sin \theta \sin \phi+\cos \psi \cos \theta & \sin \psi \sin \theta \cos \phi-\cos \psi \sin \phi \\ -\sin \theta & \cos \theta \sin \phi & \cos \theta \cos \phi\end{array}\right]$

There are two kinds of movements when the robot is stepping over the obstacles: One is translational motion; the other is rotational motion. The theoretical basis for modeling these two motions is Newton-Euler equations

$$
\left\{\begin{array}{c}
F=m \dot{v} \\
M=\dot{H}
\end{array}\right.
$$

Among it:

F_ - the summary of the external forces received by the robot

$\mathrm{v}$ - the velocity of the robot

$\mathrm{M}$ - the summary of the moment of forces received by the robot
$\mathrm{H}$ - the moment of momentum of the robot relative to the ground coordinates system

Assume the velocity of propeller is $\Omega$ and the lift force generated by the propeller is indirect proportion to the square of its rotation velocity. Then the lift force generated by the propeller is $F_{i}=b \Omega_{i}^{2}$ and the summary lift force during the obstacle crossing is

$$
\mathrm{U}=\sum_{i=1}^{4} F_{i}=F_{1}+F_{2}+F_{3}+F_{4}=b\left(\Omega_{1}^{2}+\Omega_{2}^{2}+\Omega_{3}^{2}+\Omega_{4}^{2}\right)
$$

And then the stress vector of the robot in the basic coordinates B is

$$
\mathrm{F}_{B}=\left[\begin{array}{lll}
0 & F_{\text {rope }} U
\end{array}\right]^{T}
$$

Among it, $F_{\text {rope }}$ is the tractive effort of the steel rope.

The stress vector $\mathrm{F}_{E}$ in the ground coordinates is

$$
\begin{gathered}
\mathrm{F}_{E}=\left[\begin{array}{l}
F_{X} \\
F_{Y} \\
F_{Z}
\end{array}\right]=R * F_{B}= \\
{\left[\begin{array}{c}
(\cos \psi \sin \phi-\sin \psi \cos \theta) * F_{\text {rope }}+(\cos \psi \sin \theta \cos \phi+\sin \psi \sin \phi) * \mathrm{U} \\
(\sin \psi \sin \theta \sin \phi+\cos \psi \cos \theta) * F_{\text {rope }}+(\sin \psi \sin \theta \cos \phi-\cos \psi \sin \phi) * \mathrm{U}
\end{array}\right]} \\
\cos \theta \sin \phi F_{\text {rope }}+\cos \theta \cos \phi \mathrm{U}
\end{gathered}
$$

If we assume the resistance coefficient of the robot in $\mathrm{x}, \mathrm{y}, \mathrm{z}$ coordinate axes are $\mathrm{k}_{\mathrm{x}}, \mathrm{k}_{\mathrm{y}}, \mathrm{k}_{\mathrm{z}}$ respectively. The stress equation of the robot in the three-axis ground coordinates system according to Newton's second law is

$$
\mathrm{m}\left[\begin{array}{c}
\ddot{x} \\
\ddot{y} \\
\ddot{z}
\end{array}\right]=\left[\begin{array}{c}
F_{X}-k_{x} * \dot{x} \\
F_{Y}-k_{y} * \dot{y}-m g+F_{\text {Rope }} \\
F_{Z}-k_{z} * \dot{z}
\end{array}\right]
$$

A force opposite to the rotation direction of the propeller will be formed during the propeller rotating because of air resistance, and this force will generate the yawing moment. If yaw happens to the robot, a moment of force will be generated between the steel rope and the mass center of the robot, and this moment force is so bigger than the yawing moment that the yawing moment can be neglected. There are still rolling moment and pitch moment generated when the robot is stepping over the obstacles. If we assume the yawing moment $\mathrm{Q}_{3}=0$, pitch moment is $\mathrm{Q}_{2}$, rolling moment is $\mathrm{Q}_{1}$ and the distance between the paddle center and robot center is $l$, then:

$\left[\begin{array}{l}\mathrm{Q}_{1} \\ \mathrm{Q}_{2} \\ \mathrm{Q}_{3}\end{array}\right]=l\left[\begin{array}{c}F_{4}+F_{3}-F_{2}-F_{1} \\ F_{3}+F_{2}-F_{1}-F_{4} \\ 0\end{array}\right]=l b\left[\begin{array}{c}\Omega_{4}^{2}+\Omega_{3}^{2}-\Omega_{2}^{2}-\Omega_{1}^{2} \\ \Omega_{3}^{2}+\Omega_{2}^{2}-\Omega_{4}^{2}-\Omega_{1}^{2} \\ 0\end{array}\right]$

The modeling creation of the rotational motion of the robot is mainly based on the law of conservation of angular momentum. If we assume the resultant moments received by the robot around the $x, y$, and $z$ axis are $M_{\phi}, M_{\theta}$, and $M_{\psi}$ respectively, and the rotational resistance coefficients are $k_{\phi}$, $\mathrm{k}_{\theta}$ and $\mathrm{k}_{\psi}$ respectively. Because the robot structure is geometrically symmetry, the inertia tensor of the robot is can be defined as diagonal matrix $I=\operatorname{diag}\left\{\mathrm{I}_{\mathrm{X}}, \mathrm{I}_{\mathrm{Y}}, \mathrm{I}_{\mathrm{Z}}\right\}$, then we can get the torque equilibrium equation in three coordinate axis, according to Euler equations $\mathrm{M}=\dot{\mathrm{H}}$ below:

$$
\left[\begin{array}{c}
\ddot{\phi} \\
\ddot{\theta} \\
\ddot{\psi}
\end{array}\right]=\left[\begin{array}{c}
M_{\phi} / I_{X} \\
M_{\theta} / I_{Y} \\
M_{\psi} / I_{Z}
\end{array}\right]=\left[\begin{array}{c}
\left(Q_{1}-k_{\phi} * \dot{\phi}\right) / I_{X} \\
\left(Q_{2}-k_{\theta} * \dot{\theta}\right) / I_{Y} \\
\left(Q_{3}-k_{\psi} * \dot{\psi}\right) / I_{Z}
\end{array}\right]
$$


The kinetic analysis on the robot itself can tell us the relation between the force and posture during the obstacle crossing of the robot, which can help us to generate a proper control strategy to make the obstacle crossing more stable and more efficient.

\section{Motion control design and Adams simulation}

\subsection{Motion control design}

The control system of glass-curtain wall-cleaning robot is composed of control segment of the robot itself, wireless communication, human-machine interaction and auxiliary motion control module on the building roof. Constant voltage source will supply power to the control segment in the robot, the cleaning roller in the cleaning mechanism will be driven by the DC-motor and there is also temperature protection, over current protection and low voltage protection modules in the control segment of the robot itself. Lots of sensors are equipped in the control segment and used to detect the posture of the robot and the window frame. The pressure sensor can measure the pressure between the cleaning mechanism and glass surface. The STM32 master board will realize the robot control and data transmission. The human-machine interaction part will make the human intervention and control more efficient. The Figure 7 has shown the overall control system of the cleaning robot.

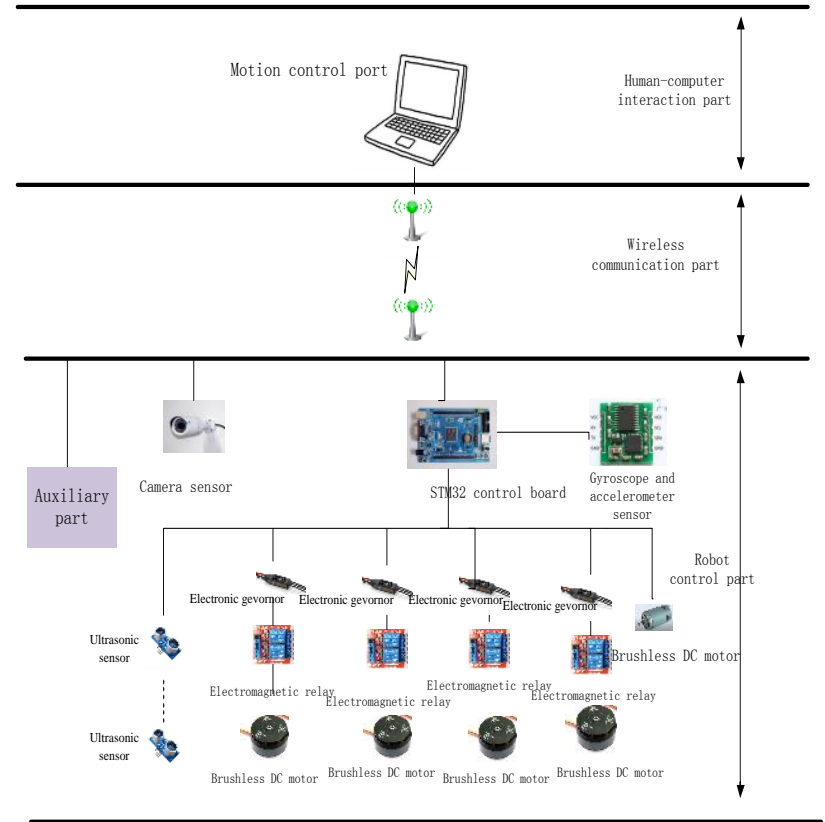

Figure 7: Sketch map of overall control system of the cleaning robot

The robot will mainly adopt the autonomous operation working mode. The gyroscope sensor installed in the robot can detect the operating posture and speed of the robot and the robot posture can be adjusted by the rotation speed of propeller, so that the pressure on different cleaning mechanism is roughly equal and the cleaning effect will be better. When the visual sensor has detected the window frame, the image information of the frame will be transmitted back to
PC through wireless communication. After the PC finishes the process of the image, the obstacle crossing program will be started according to the obstacle height. The block diagram of autonomous control program of the robot is shown in Figure 8.

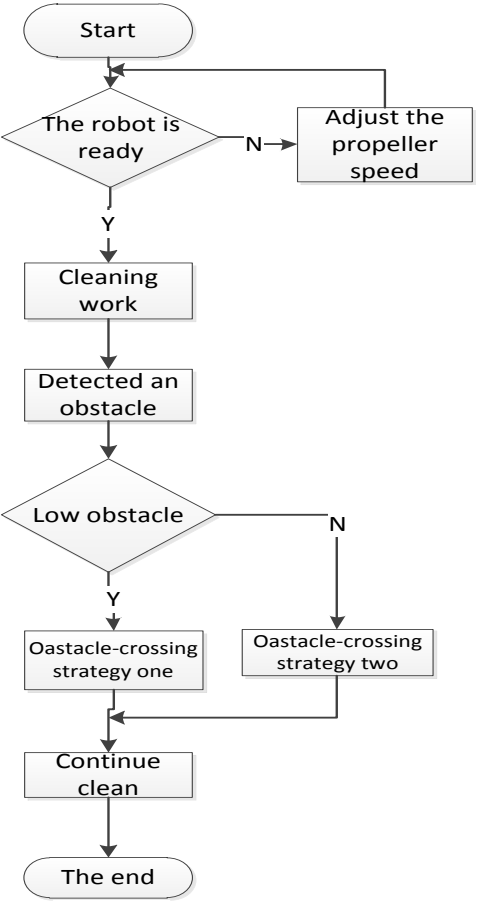

Figure 8: The block diagram of autonomous control program of the robot

\subsection{Adams simulation about the obstacle crossing process}

This thesis makes an Adams emulation analysis on the situation that the cleaning robot steps over a higher obstacle. Below is the emulation process in detail: The robot accelerates from static to the velocity $\mathrm{v}_{0}$ in vertical direction, before it comes across the obstacle and then keep working with the speed of $\mathrm{v}_{0}$. when the robot detects the frame, it starts to slow down and the vertical speed decreases to 0 when it come to the frame. The obstacle crossing process will be carried out in three phases: First the rotating direction of the propeller will be change which makes the velocity in horizontal direction change from 0 to constant velocity $v_{1}$. After a while it starts deceleration motion. After it lift over the window frame, the horizontal velocity will decrease to 0 . Second, the robot will accelerate its velocity in vertical from 0 to $v_{2}$ and to keep the constant velocity motion and then slow down the velocity to 0 after it moves to the height lower than the window frame. The third phase is opposite to the first phase, and the robot accelerates from 0 to $\mathrm{v}_{0}$ and continue to clean. Figure 9a shows the velocity curve in vertical direction during the whole cleaning process, figure $9 \mathrm{~b}$ shows the acceleration curve in vertical direction, figure $9 \mathrm{c}$ shows the velocity curve in horizontal direction and figure $9 \mathrm{~d}$ shows acceleration curve in horizontal direction. 


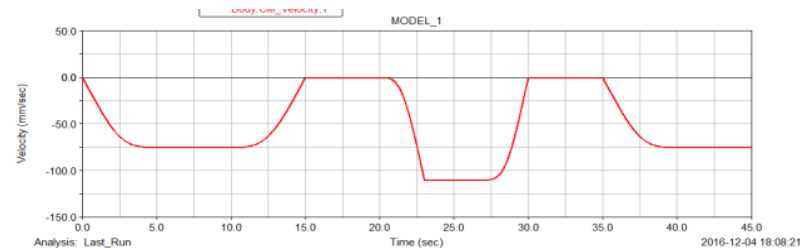

a: The velocity curve in vertical direction

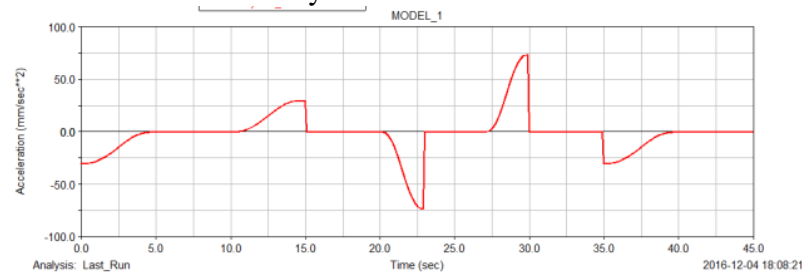

$\mathrm{b}$ : The acceleration curve in vertical direction

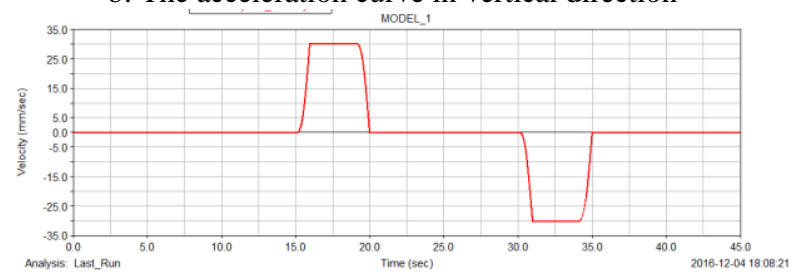

c: The velocity curve in horizontal direction

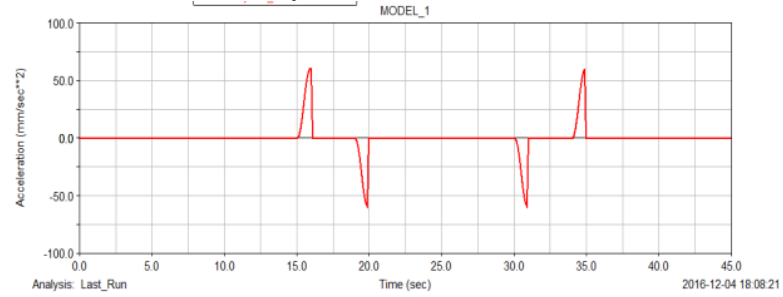

$\mathrm{d}$ : The acceleration curve in horizontal direction

Figure 9: the Adam simulation results

From the simulation analysis, we can see the velocity in the whole process is stable, without obvious jumping. The emulation data can be used as a reference to the design of the real control system.

\section{Conclusion}

With the adoption of the increasing adoption of glass curtain wall in the high building, the task to clean these glass curtain wall is becoming more and more heavier. And traditional manual cleaning is dangerous and of low efficient. The cleaning robot has solved these problems. However, the vacuum sucking disc based on the negative pressure has an extremely high requirement on the air tightness and magnetic adsorption requires a magnetic surface. The applicability of above solutions is not good enough. The propellant attaching introduced in this paper has improved the adaptive capacity about the wall surface. Additionally, the working efficiency has been lifted up through the way that the robot can execute different obstacle crossing function according to different obstacles. What's more, the simulation of the obstacle crossing process has been done with the software Adams, providing a reference to the actual control system design.

\section{References}

[1] Zhang H, Zhang J, Zong G. Realization of a Service Climbing Robot for Glass-wall Cleaning[C]// IEEE
International Conference on Robotics and Biomimetics. IEEE, 2004:395-400.

[2] Bahr B, Wu F, Bahr B, et al. Design and safety analysis of a portable climbing robot[C]// International Conference on Robotics \& Automation. 1994.

[3] Nishi A, Miyagi H. Mechanism and Control of Propeller Type Wall-Climbing Robot[C]// International Conference on Intelligent Robots and Systems '94. 'advanced Robotic Systems and the Real World', IROS. 1994:669-677.

[4] Galt S, Luk B L, Cooke D S, et al. A tele-operated semi-intelligent climbing robot for nuclear applications $[\mathrm{C}] / /$ Conference on Mechatronics and Machine Vision in Practice. IEEE Computer Society, 1997:118-118.

[5] Hirose S, Aoki S. Development of the Insulator Washer robot[J]. 1995, 2:1783-1789 vol.2.

[6] Zhao Yanzheng, Men Guangliang Feng Mei etc. Research on Posture Control of the All-around Wall Climbing Robot [J]. JOURNAL OF HARBIN INTSITUTE TECHNOLOGY, 1997(06):116-122.

[7] Zhang H, Zhang J, Wang W, et al. A series of pneumatic glass - wall cleaning robots for high - rise buildings[J]. Industrial Robot, 2007, 34(2):150-160.

[8] Tan Lishi. Design Analysis and simulation of the System of Wall Climbing Robot [D]. Shanghai University of Science and Technology, Shanghai University, 1991.

[9] Qian Ziyuan, Fu Z, et al. Design and realization of a non-actuated glass-curtain wall-cleaning robot prototype with dual suction cups[J]. The International Journal of Advanced Manufacturing Technology, 2006, 30(1):147-155.

[10] Zhu J, Sun D, Tso S K. Application of a Service Climbing Robot with Motion Planning and Visual Sensing[J]. Journal of Robotic Systems, 2003, 20(4):189-199.

[11] Xiao J, Bing L I, Ushiroda K, et al. RISE-ROVER:A Wall-Climbing Robot With High Realiability And Load Carrying Capacity[M]// Assistive Robotics. 2015:784.

[12] Tan Guangchao, Design and Implementation of Attitude Control System for A Quadrotor UAV [D]. Dalian University of Technology, 2013. 